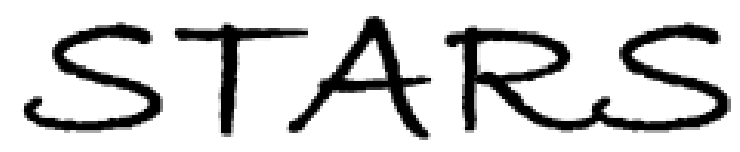

University of Central Florida

STARS

$1-1-2004$

\title{
Tunable diffraction grating using ultraviolet-light-induced spatial phase modulation in dual-frequency liquid crystal
}

\author{
Pao-Tai Lin \\ University of Central Florida \\ Xiao Liang \\ University of Central Florida \\ Hongwen Ren \\ University of Central Florida \\ Shin-Tson Wu \\ University of Central Florida
}

Find similar works at: https://stars.library.ucf.edu/facultybib2000

University of Central Florida Libraries http://library.ucf.edu

This Article is brought to you for free and open access by the Faculty Bibliography at STARS. It has been accepted for inclusion in Faculty Bibliography 2000s by an authorized administrator of STARS. For more information, please contact STARS@ucf.edu.

\section{Recommended Citation}

Lin, Pao-Tai; Liang, Xiao; Ren, Hongwen; and Wu, Shin-Tson, "Tunable diffraction grating using ultravioletlight-induced spatial phase modulation in dual-frequency liquid crystal" (2004). Faculty Bibliography 2000s. 4544.

https://stars.library.ucf.edu/facultybib2000/4544

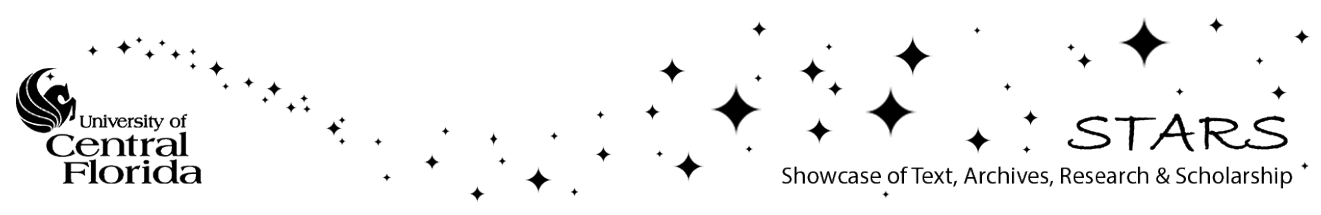




\section{Tunable diffraction grating using ultraviolet- light-induced spatial phase modulation in dual-frequency liquid crystal}

Cite as: Appl. Phys. Lett. 85, 1131 (2004); https://doi.org/10.1063/1.1781752

Submitted: 12 April 2004 . Accepted: 22 June 2004 . Published Online: 10 August 2004

Pao-Tai Lin, Xiao Liang, Hongwen Ren, and Shin-Tson Wu

\section{ARTICLES YOU MAY BE INTERESTED IN}

Fast switching dual-frequency liquid crystal optical retarder, driven by an amplitude and frequency modulated voltage

Applied Physics Letters 83, 3864 (2003); https://doi.org/10.1063/1.1625114

Polarization-independent and submillisecond response phase modulators using a $90^{\circ}$ twisted dual-frequency liquid crystal

Applied Physics Letters 89, 021103 (2006); https://doi.org/10.1063/1.2219998

Optical diffraction from a liquid crystal phase grating

Journal of Applied Physics 91, 3495 (2002); https://doi.org/10.1063/1.1446216

\section{Applied Physics Letters}

Mid-IR and THz frequency combs special collection

\section{Read Now!}




\title{
Tunable diffraction grating using ultraviolet-light-induced spatial phase modulation in dual-frequency liquid crystal
}

\author{
Pao-Tai Lin, Xiao Liang, Hongwen Ren, and Shin-Tson Wua) \\ College of Optics and Photonics, University of Central Florida, Orlando, Florida 32816
}

(Received 12 April 2004; accepted 22 June 2004)

\begin{abstract}
An electrically tunable diffraction phase grating using ultraviolet (UV)-light-induced spatial dielectric modulation of a dual-frequency liquid crystal (DFLC) cell is demonstrated. A photomask with transparent and opaque stripes was used for fabricating the grating. In the UV-exposed stripes, the negative dielectric anisotropy $(\Delta \varepsilon)$ tolane compound of the DFLC mixture is partially polymerized resulting in a decreased threshold voltage as compared to that of the unexposed region. Upon applying a constant voltage, the phase difference between the adjacent pixels is produced. The first-order diffraction efficiency reaches $\sim 60 \%$ which agrees well with the simulation results. Due to the dual-frequency addressing at $30 V_{\text {rms }}$, the response time of the DFLC phase grating was measured to be $\sim 1 \mathrm{~ms}$ at room temperature. (C) 2004 American Institute of Physics.
\end{abstract}

[DOI: $10.1063 / 1.1781752]$

Liquid crystal (LC) phase grating has been widely used for laser beam steering, ${ }^{1}$ tunable-focus lens, ${ }^{2}$ and foveated imaging. ${ }^{3}$ Two physical mechanisms have been commonly employed for demonstrating phase grating devices: A homogeneous LC layer with an inhomogeneous electric field, and an inhomogeneous LC layer with a homogeneous electric field. The former examples include optical phased arrays (OPAs) and the latter include alternative twisted-nematic domains, ${ }^{4}$ modulated Freederisckz transition threshold in dual-frequency LC material, ${ }^{5}$ and gradient refractive index nanoscale polymer-dispersed liquid crystal (GRIN PDLC) droplets. ${ }^{6}$ Each approach has its own pros and cons. An OPA exhibits a high diffraction efficiency and low operating voltage, however, its pixilated structure is sophisticated. On the other hand, the GRIN PDLC has a fast response time and is independent of polarization except that its phase change is small and the operating voltage is high $\left(>100 V_{\text {rms }}\right)$. There is an urgent need to develop phase gratings with high diffraction efficiency, fast response time, and low operating voltage.

In this letter, we demonstrate a phase grating based on the spatial dielectric modulation of the dual-frequency liquid crystal (DFLC). For a DFLC, the dielectric anisotropy $(\Delta \varepsilon)$ is positive at low frequencies, gradually decreases to zero, and then becomes negative as the frequency increases. ${ }^{7}$ The frequency that $\Delta \varepsilon=0$ is called cross-over frequency $\left(f_{c}\right)$. In practice, a DFLC mixture is comprised of positive and negative $\Delta \varepsilon$ LC compounds and its cross-over frequency is around a few kilohertz, depending on the molecular structures and compositions. The major attraction of the DFLC device is fast response time. During the turn-on and -off processes, ac voltage bursts with low and high frequencies are applied. As a result, fast rise and decay times are achieved. In our device, the phase grating is controlled by the UV exposure through a spatially alternating opaque and transparent photomask. With this simple design, the measured first-order $( \pm 1)$ diffraction efficiency achieves $\sim 60 \%$.

To fabricate the LC phase grating, we exposed UV ( $\lambda$ $\sim 365 \mathrm{~nm}$ ) light to the DFLC cell through a patterned pho-

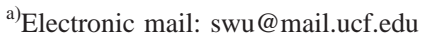

tomask. The width of the alternating transparent stripe is $16 \mu \mathrm{m}$ and the opaque stripe is $234 \mu \mathrm{m}$. Figure 1(a) depicts the experimental setup. The photomask is in proximity contact with the LC cell. A high performance DFLC mixture consisting of biphenyl esters $(\Delta \varepsilon \sim 30)$ and laterally difluorotolanes $(\Delta \varepsilon \sim-6)$ has been recently reported. ${ }^{8}$ For proving principle, we used a binary DFLC mixture consisting of $20 \%$ biphenyl ester and $80 \%$ difluoro-tolane. Its cross-over frequency occurs at $f_{c} \sim 1.1 \mathrm{kHz}$. The binary DFLC mixture was injected into a homogeneous cell with gap $d=7.7 \mu \mathrm{m}$ by capillary flow. The transmitted UV light through the photomask generates free radicals around the carbon-carbon triple bonds of the difluoro tolane molecules and causes a partial polymerization. ${ }^{9}$ On the other hand, the biphenyl esters are inert to the UV irradiation because of their shorter molecular conjugation. The small reduction of the difluoro tolane composition causes the cross-over frequency to increase because

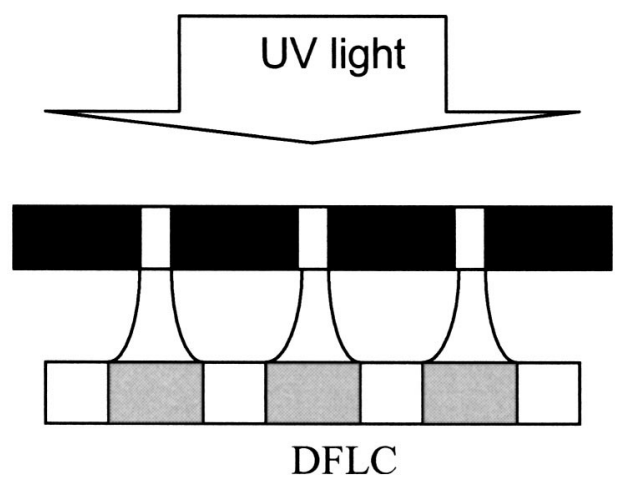

(a)

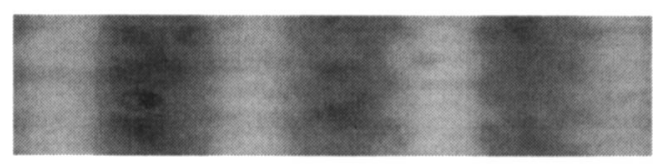

(b)

FIG. 1. (a) Experimental setup for fabricating DFLC phase grating. (b) Microscope image of the UV exposed DFLC through photomask. Polarizers are crossed. 


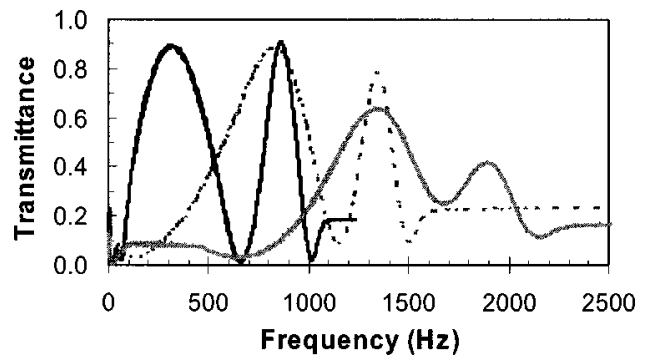

FIG. 2. Frequency-dependent transmittance of three DFLC cells with 0 (dark line), $200 \mathrm{~s}$ (gray), and $300 \mathrm{~s}$ (thin dark line) UV exposure time, respectively. The applied voltage is fixed at $15 V_{\text {rms }} ; T=23{ }^{\circ} \mathrm{C}$ and $\lambda$ $=633 \mathrm{~nm}$.

the mixture's $\Delta \varepsilon$ becomes more positive in the lowfrequency regime. Therefore, the exposed pixels exhibit a lower threshold voltage than the opaque ones. If we apply a constant low-frequency voltage to the DFLC cell, the transparent and opaque stripes will have a different phase difference. As a result, the diffraction effect would occur.

Figure 1(b) shows the microscope image of the exposed DFLC cell situated between crossed polarizers. Indeed, the exposed and unexposed stripes have different phase retardations. The transmitted UV light from the photomask is diffracted and after passing the top 1.1-mm-thick glass substrate, the beam is expanded by $\sim 5 \times .{ }^{10}$ From Fig 1(b), the ratio of the unexposed and exposed stripes is $\alpha \sim 0.6$. This parameter will be used later for calculating the diffraction efficiency.

To examine how UV affects the cross-over frequency, we compare three DFLC samples; A, B, and C which correspond to 0,200 , and $300 \mathrm{~s}$ of UV exposures. In this study, no photomask was used. Figure 2 shows the frequencydependent transmittance of these three samples. The applied voltage was fixed at $V=15 V_{\text {rms }}$ and the transmittance measured with a He-Ne laser $(\lambda=633 \mathrm{~nm})$ between two crossed polarizers at $T \sim 23{ }^{\circ} \mathrm{C}$. In the low-frequency regime, the DFLC has a positive $\Delta \varepsilon$ so that the LC directors are reoriented by the electric field. As the frequency approaches $f_{c}$, the dielectric anisotropy gradually decreases which implies to an increased threshold voltage. In the $f \geqslant f_{c}$ regime, the low-frequency electric field can no longer reorient the LC directors because $\Delta \varepsilon<0$. As a result, the transmittance remains flat. From Fig. 2, the cross-over frequency of the unexposed sample (dark line) is $f_{c} \sim 1.1 \mathrm{kHz}$. As UV exposure time increases to $200 \mathrm{~s}$ (dashed lines) and $300 \mathrm{~s}$ (gray line), the cross-over frequency increases to 1.65 and $2.3 \mathrm{kHz}$, respectively. Due to this frequency shift, at $V=15 V_{\mathrm{rms}}$ and $f$ $=1 \mathrm{kHz}$, the phase difference between samples $\mathrm{A}$ and $\mathrm{C}$ is $\delta \sim 3.7 \pi$. This experiment proves that the phase difference between the exposed and unexposed areas can be controlled by the UV intensity and the applied voltage and frequency.

Based on the results obtained from Fig. 2, we fabricated a phase grating using a photomask shown in Fig. 1(a). At $V=30 V_{\text {rms }}$, the DFLC gratings have the highest diffraction efficiency. Since the homogeneous LC cell is uniaxial, only the extraordinary ray experiences the spatial phase retardation during molecular reorientation. Figure 3 plots the frequency-dependent normalized diffracted laser power of the DFLC samples with $200 \mathrm{~s}$ (left) and $300 \mathrm{~s}$ (right) UV exposures through photomask. Here, unity stands for the transmittance at $V=0$ where no diffraction occurs. The UV exposure time not only affects the cross-over frequency but

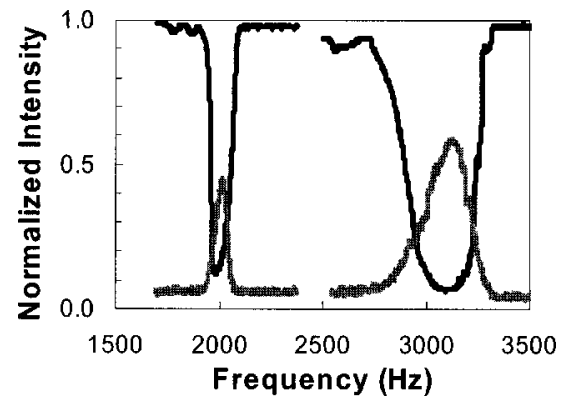

FIG. 3. Frequency-dependent diffracted HeNe laser power of the DFLC cell after $200 \mathrm{~s}$ (left) and $300 \mathrm{~s}$ (right) UV exposure through a photomask. The applied voltage is fixed at $30 V_{\mathrm{rms}} ; T=23{ }^{\circ} \mathrm{C}$ and $\lambda=633 \mathrm{~nm}$. Gray lines represent first-order diffractions.

also broadens its bandwidth. As the UV exposure increases from 200 to $300 \mathrm{~s}$, more difluoro-tolane molecules are polymerized in the exposed stripes so that the cross-over frequency shifts from $\sim 1.9 \mathrm{kHz}$ to $2.75 \mathrm{kHz}$. Moreover, its bandwidth is widened from $\sim 200 \mathrm{~Hz}$ to $\sim 500 \mathrm{~Hz}$. The measured first-order peak diffraction efficiency (gray lines) increases from $45 \%$ to $59 \%$. For a longer UV exposure, more tolane molecules are polymerized and the LC alignment in the exposed pixels is disturbed. The former factor leads to an enlarged phase difference between the adjacent pixels and the latter suppresses the higher diffraction orders. The overall effect is that the first-order diffraction efficiency is improved. The response time of the DFLC gratings $(d=7.7 \mu \mathrm{m})$ with $200 \mathrm{~s}$ and $300 \mathrm{~s}$ UV exposures were measured to be $\sim 1 \mathrm{~ms}$ and $2 \mathrm{~ms}$, respectively, when the frequency is switched from $f_{c}$ to $50 \mathrm{kHz}$ while keeping voltage at $V=30 V_{\mathrm{rms}}$. As the voltage increases, the response time is faster, however, the diffraction efficiency gradually decreases.

The observed fast response is the key feature of a DFLC device. In the low-frequency regime, the DFLC has a positive $\Delta \varepsilon$. The applied voltage (say $30 V_{\text {rms }}$ ) reorients the LC directors from a homogeneous to a homeotropic direction. During decay process, a high-frequency voltage is applied. Under such a circumstance, the $\Delta \varepsilon$ becomes negative so that the applied electric field exerts a torque to assist the LCs relax back from a homeotropic to a homogeneous direction.

Figure 4 shows the diffraction patterns captured by a camera. The lower photo in Fig. 4(a) shows the input light while the top photo shows the diffraction patterns for the sample with $200 \mathrm{~s}$ UV exposure. Up to \pm fourth-diffraction orders are observed as the frequency gets close to $f_{c}$. Outside this region, diffraction does not occur. When $f>f_{c}$, the LC molecules do not respond to the electric field because $\Delta \varepsilon$ is negative. In the low-frequency regime where $f \ll f_{c}$, diffraction also disappears because all the LCs (no matter in the opaque or transparent stripes) are all reoriented by the electric field even though their threshold voltage is somewhat different. The phase difference between the adjacent pixels is so small that the phase grating vanishes. Figure 4(b) shows the diffraction patterns for the DFLC cell with $300 \mathrm{~s}$ UV exposure. The diffraction intensity is strong for the zeroth, \pm first, and \pm second orders and vanishes quickly after the third order. Comparing Figs. 4(a) and 4(b), we find that the higher-order patterns are more noticeable for the shorter UV exposed cell. By contrast, diffraction concentrates in the lower orders for the cell with a longer UV exposure.

To understand the physical mechanism of the DFLC phase grating, we have performed computer simulations of 
the device structures. For a beam passing through a uniaxial LC, the accumulated phase is related to the birefringence, molecular tilted angle, cell gap, and wavelength. For a given LC material and device, the only variable is the molecular tilt angle which is determined by the voltage. By tuning the applied voltage $V$ and frequency $f$ of the electric field, the LC director reorientation can be calculated. For a LC phase grating, it is necessary to calculate the spatial phase difference. After a laser beam traverses a homogeneous LC cell, the phase retardation $\delta$ (in the small angle regime) is equal to ${ }^{11}$

$$
\delta=\delta_{\max } \frac{\left[\left(\frac{n_{e}}{n_{o}}\right)^{2}+\left(\frac{n_{e}}{n_{o}}\right)\right]}{\left[\frac{k_{33}}{k_{11}}+\frac{\Delta \varepsilon}{\left(\varepsilon_{\perp}\right)_{i}}\right]}\left(\frac{V}{\pi \sqrt{\frac{K_{i i}}{\varepsilon_{o} \Delta \varepsilon_{i}}}}-1\right) .
$$

Here, $i=1$ stands for the unexposed pixels and $i=2$ for the exposed ones. If the spatial variation of $\Delta \varepsilon(f)$ is considered, the amplitude distribution $U$ with phase difference $\phi$ between the opaque and transparent pixels can be described as: ${ }^{12}$

$$
\begin{aligned}
U= & \left\{\operatorname{rect}\left(\frac{x-\frac{(\alpha)}{2} P}{(\alpha) P}\right)\right. \\
& \left.\times e^{-i \phi}+\operatorname{rect}\left(\frac{x-\frac{(1-\alpha)}{2} P}{(1-\alpha) P}\right)\right\} \cdot \frac{1}{P} \operatorname{comb}\left(\frac{x}{P}\right),
\end{aligned}
$$

where $P$ is the period of the photomask and $\alpha$ is the percentage of the opaque area of the grating. Taking the Fourier transform of Eq. (2), the diffraction efficiency $(\eta)$ of the $n$th order has the following form:

$$
\eta=\sqrt{\alpha^{2} \sin c^{2}(n \alpha)+(1-\alpha)^{2} \sin c^{2}[n(1-\alpha)]+2 \alpha(1-\alpha) \sin c(n \alpha) \sin c[n(1-\alpha)] \cos (\phi-n \pi)} .
$$

From Fig. 1(b), we find $\alpha \sim 0.6$. Under such a condition, the peak diffraction efficiency reaches $\eta \sim 0.6$, which agrees well with the experimental data $(\eta \sim 0.59)$. From theory, the highest diffraction efficiency occurs at $\alpha=0.5$, i.e., equal
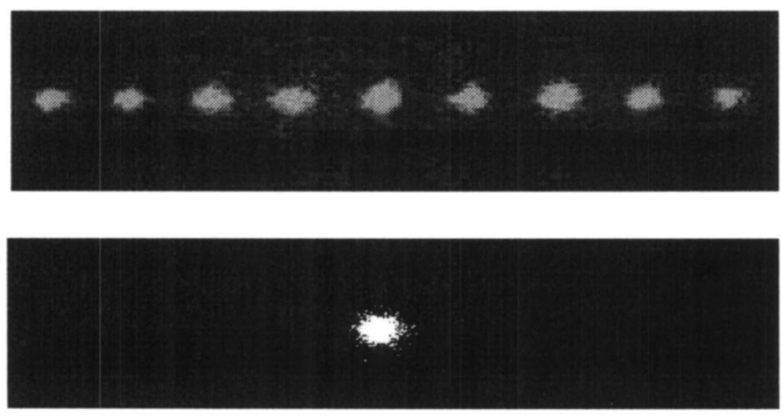

(a) $200 \mathrm{~s}$
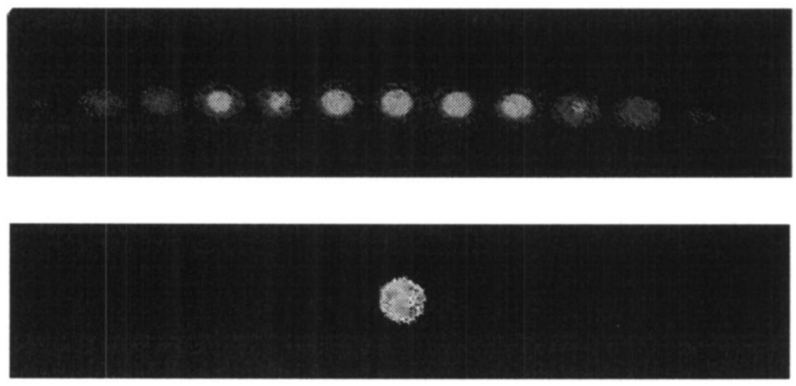

(b) $300 \mathrm{~s}$

FIG. 4. Diffraction patterns of the DFLC cell after (a) $200 \mathrm{~s}$ and (b) $300 \mathrm{~s}$ UV exposure. The UV illumination is through a photomask. The single spot photos represent the nondiffraction stage at $f>f_{c}$. The applied voltage is fixed at $30 V_{\text {rms }} ; T=23^{\circ} \mathrm{C}$ and $\lambda=633 \mathrm{~nm}$. opaque and transparent pixel size. This result is important for designing the photomask patterns for optimizing the grating performance.

In conclusion, we demonstrate a frequency controlled diffraction phase grating based on the selected UV-induced polymerization in a DFLC cell. The measured first-order diffraction efficiency reaches $\sim 60 \%$. Simulation results agree well with experiments. Potential applications of such a device are for laser beam steering and an imaging system where low cost, low applied voltage, and fast response time are critical.

This work is supported by AFOSR under Contract No. F49620-01-1-0377.

${ }^{1}$ P. F. McManamon, T. A. Dorschner, D. L. Corkum, L. Friedman, D. S. Hobbs, M. Holz, S. Liberman, H. Q. Nguyen, D. P. Resler, R. C. Sharp, and E. A. Watson, Proc. IEEE 84, 268 (1996).

${ }^{2}$ H. Ren, Y. H. Fan, and S. T. Wu, Appl. Phys. Lett. 82, 3168 (2003).

${ }^{3}$ D. V. Wick, T. Martinez, S. R. Restaino, and B. R. Stone, Opt. Express 10, 60 (2002).

${ }^{4}$ C. M. Titus and P. J. Bos, Appl. Phys. Lett. 71, 2239 (1997).

${ }^{5}$ H. Ren and S. T. Wu, Appl. Phys. Lett. 81, 3537 (2002).

${ }^{6}$ S. W. Kang, S. Sprunt, and L. C. Chien, Appl. Phys. Lett. 78, 3782 (2001).

${ }^{7}$ H. K. Bucher, R. T. Klingbiel, and J. P. VanMeter, Appl. Phys. Lett. 25, 186 (1974).

${ }^{8}$ J. Thisayukta, T. Masumi, Y. Shiraishi, N. Toshima, S. Kobayashi, A. Sawada, and S. Naemura, Soc. Information Display Tech. Digest 34, 696 (2003).

${ }^{9}$ P. T. Lin and S. T. Wu, Mol. Cryst. Liq. Cryst. 411, 243 (2004).

${ }^{10}$ H. Ren, Y. H. Fan, S. Gauza, and S. T. Wu, Opt. Commun. 230, 267 (2004).

${ }^{11}$ I. C. Khoo and S. T. Wu, Optics and Nonlinear Optics of Liquid Crystals (World Scientific, Singapore, 1993), Chap. 2.

${ }^{12}$ J. W. Goodman, Introduction to Fourier Optics, 2nd ed. (McGraw-Hill, New York, 1996), Chap. 3. 